\title{
Orale und systemische Erkrankungen. Teil 1: Eine auf Evidenz basierende Übersicht ihrer Wechselwirkungen
}

\author{
Christian E. Besimo \\ Abteilung Zahnmedizin, Aeskulap-Klinik, Brunnen, Schweiz
}

wischen oralen und systemischen Erkrankungen gibt es grundsätzlich vier mögliche Wechselbeziehungen [1]:

1. Pathologische Veränderungen im Zahn-, Mund- und Kieferbereich oder zahnärztliche Therapiemassnahmen können allgemeinmedizinische Erkrankungen auslösen bzw. beeinflussen (z. B. bakterielle Endokarditis).

2. Umgekehrt ist es aber auch möglich, dass Allgemeinerkrankungen Veränderungen im Kauorgan verursachen bzw. Auswirkungen haben auf den Verlauf oraler Erkrankungen (z. B. Lupus erythematodes, Morbus Crohn).

3. Zudem können internistische Erkrankungen zu Komplikationen bei der zahnärztlichen Therapie führen (z. B. hypertensive Krisen bei arterieller Hypertonie).

4. Schliesslich muss vergleichbar mit Medikamenten auch bei der Verwendung zahnärztlicher Werkstoffe immer mit Nebenwirkungen gerechnet werden.

\section{Mögliche Zusammenhänge zwischen oralen und syste- mischen Erkrankungen}

Mögliche Zusammenhänge zwischen oralen und allgemeinen Erkrankungen sind in zunehmendem Masse Gegenstand wissenschaftlicher Untersuchungen [2,3]. Ein intensiv studiertes Modell stellt dabei die marginale Parodontitis dar. Diese wird heute, wenn auch kontrovers, als Risikofaktor für

Mögliche Zusammenhänge zwischen oralen und allgemeinen Erkrankungen sind in zunehmendem Masse Gegenstand wissenschaftlicher Untersuchungen. Die nachfolgende kurze Literaturübersicht und die Patientenbeispiele verdeutlichen einerseits, dass nicht nur grössere, sondern auch kleinere orale Infekte andere Erkrankungen des Organismus negativ beeinflussen bzw. deren medizinische Therapie in ihrer Wirksamkeit beeinträchtigen können. Sie unterstreichen andererseits auch die Notwendigkeit einer bisher nach wie vor vernachlässigten engen interdisziplinären Vernetzung der medizinisch-zahnärztlichen Diagnostik und Therapie.

Schlüsselwörter: Orale Erkrankungen, systemische Erkrankungen, Wechselwirkungen, Parodontitis

\section{Oral and Systemic Diseases \\ Part 1: An Evidence-based Review of their Interrelationships}

Possible connections between oral and systemic diseases are increasingly a topic of scientific research. The present short review of the literature and the clinical cases reveals that not only more severe but also minor oral infections can have a negative impact on systemic diseases and on the efficacy of their medical therapy. They underline, moreover, the need for so far little appreciated close cooperation and interdisciplinary communication to create a system of medicodental diagnostics and therapy.

Key words: Oral diseases, systemic diseases, interactions, periodontitis

Herz- und Kreislauferkrankungen diskutiert [4-17]. Zahlreiche epidemiologische Studien und tierexperimentelle Untersuchungen weisen darauf hin, dass eine pathogenetische Beziehung zwischen marginaler Parodontitis und kardiovaskulären Erkrankungen besteht. Dies betrifft insbesondere die koronare Herzkrankheit, Schlaganfall und periphere arterielle Durchblutungsstörungen. Dabei wird die systemische Belastung durch orale Mikroorganismen und Entzündungsfaktoren infolge parodontaler Erkrankungen als bedeutsam angesehen. Die Erkenntnislage scheint ausreichend $\mathrm{zu}$ sein, um parodontale Erkrankungen in die Checkliste zur Erstellung des Risikoprofils für koronale Herzerkrankungen und Schlaganfall aufzunehmen bzw. bei positivem Risikobefund konsequent zu behandeln [18-22].

\section{Rheumatoide Arthritis}

Ähnlichkeiten der Pathogenese bestehen auch zwischen rheumatoider Arthritis und Parodontitis. Diese weisen auf mögliche Zusammenhänge zwischen Schweregrad der parodontalen und Ausprägung der artikulären Entzündung hin [23,24]. Wie das erste der nachfolgend beschriebenen Patienten- 
beispiele dokumentiert, können vergleichbare parodontale Einflüsse auch auf die Symptomatik degenerativer Gelenkserkrankungen beobachtet werden.

\section{Diabetes mellitus}

Es wird seit längerer Zeit eine wechselseitige Beeinflussung zwischen marginaler Parodontitis und Diabetes mellitus beschrieben [25-34]. Vergleichbare Wechselwirkungen werden auch für die apikale Parodontitis in Betracht gezogen [35]. Die marginale Parodontitis ist nicht nur selbst eine Komplikation des Diabetes mellitus, sondern trägt zudem zur Verschlimmerung der humoralen Erkrankung bei und beeinträchtigt deren therapeutische Kontrolle. Insgesamt entstehen durch den Diabetes mellitus besondere Bedingungen, die zu einer Zunahme von Entzündungen im Mundraum führen. Diese Entzündungen sind gekennzeichnet durch eine Überproduktion von inflammatorischen Mediatoren und Gewebe abbauenden Enzymen, die nicht nur den Verlauf der Parodontitis, sondern auch die Ausprägung der Rezidive signifikant verstärken. Ein schlecht eingestellter Diabetes mellitus kann zudem das Risiko für andere diabetische Komplikationen wie Angiopathie, Nephropathie, Retinopathie, Neuropathie und verzögerte Wundheilung erhöhen [36-40]. Bei Diabetes Typ-II-Patienten kann eine Verbesserung der glykämischen Kontrolle durch Behandlung der parodontalen Entzündung beobachtet werden [41-43]. Studien an Diabetes Typ-I-Patienten vermögen jedoch keine signifikante Wirkung der Parodontaltherapie auf die Stoffwechselkontrolle nachzuweisen. Dieser Unterschied der biologischen Therapieantwort dürfte auf die Entzündung zurückzuführen sein, die bei der Pathogenese des Typ-II-Diabetes eine Rolle spielt, nicht aber beim Typ-I-Diabetes, der durch eine Autoimmunzerstörung der Insulin produzierenden Zellen gekennzeichnet ist [44,45]. Eine adäquate Blutzuckereinstellung senkt nicht nur die Glukosekonzentration im Blutserum, in der Sulkusflüssigkeit der Gingiva und im Speichel, sondern vermindert auch die Entzündungsreaktion. Die Kontrolle der chronischen gramnegativen parodontalen Infektion sollte deshalb fester Bestandteil der Standardbehandlung diabetischer Patienten sein [46].

\section{Atemwegsinfektionen}

$\mathrm{Zu}$ den vom oralen Gesundheitszustand ebenfalls beeinflussten Krankheiten werden gerade bei institutionalisierten Betagten Atemwegsinfektionen, wie z. B. die Pneumonie, gezählt Die Mortalität ist bei Pneumonien im Alter infolge der geschwächten Immunabwehr deutlich erhöht. Eine Halbierung der Pneumonierate bei Pflegeheimpatienten durch Mundhygiene nach jeder Mahlzeit und regelmässige professionelle Zahnreinigung ist klinisch nachgewiesen worden. Interessanterweise ist dieser Effekt auch bei zahnlosen Patienten nachweisbar [8, 30,32,47-53].

\section{Osteoporose}

Es besteht begrenzte aber überzeugende Evidenz, dass eine Beziehung zwischen Osteoporose und parodontaler Erkrankung und Zahnverlust besteht. So finden sich Hinweise, dass die generalisierte Abnahme der Knochendichte mit einer Zunahme des parodontalen Attachment- und Zahnverlustes korreliert. Einige Studien haben zudem eine Verbesserung des parodontalen Gesundheitszustandes durch Behandlung der Osteoporose beschrieben. Es wird vermutet, dass viele der bei Osteoporose wirksamen Massnahmen, wie z. B. Pharmakotherapie, Ernährungslenkung oder Änderung der Lebensführung, auch bei der Parodontitisbehandlung erfolgreich eingesetzt werden könnten. Es bleibt abzuwarten, inwieweit die zahnärztliche Diagnostik mit der Panoramaschichtaufnahme in der Lage sein wird, unterstützend bei der Früherkennung von Osteoporose mitzuwirken [54-62]. Weitere interessante Forschungsansätze ergeben sich aus japanischen Studien, die darauf hindeuten, dass einerseits Zahnverlust im fortgeschrittenen Alter zu einer Beeinträchtigung der räumlichen Orientierung führen bzw. umgekehrt die Erhaltung einer funktionellen Okklusion zur Sturzprävention beitragen könnte $[63,64]$.

\section{Psychosozialer Stress}

Klinische Studien geben den Hinweis, dass psychosozialer Stress Entstehung und Verlauf der marginalen Parodontitis negativ zu beeinflussen vermag. Die Kombination mit anderen parodontalen Risikofaktoren, wie z. B. Nikotinabusus, kann verstärkend wirken. In diesem Zusammenhang sollte beachtet werden, dass gerade bei älteren Patienten nicht nur parodontale Erkrankungen gehäuft auftreten, sondern auch Stresssituationen [65-68]. Weiterhin wird die Parodontitis als möglicher Kofaktor für Frühgeburten und geringes Geburtsgewicht diskutiert [8, 9,69-74].

\section{Organabszesse}

Organabszesse sind eine seltene und lebensbedrohliche Komplikation. Odontogene Infektionen sind als Ursache von Hirnabszessen bekannt [75-77]. Auch Leberabszesse infolge einer Zahnbehandlung oder mit Assoziation zu einer Zahnerkrankung sind in der Literatur beschrieben [78-80]. Als orale Manifestation einer systemischen Erkrankung sind Veränderungen der Mundschleimhaut bzw. des Parodontes beispielsweise für den systemischen Lupus erythematodes und Morbus Crohn beschrieben worden [81-83].

\section{Zahnmedizinische Materialien}

Vergleichbar mit Medikamenten muss auch bei zahnärztlichen Werkstoffen immer mit Nebenwirkungen gerechnet werden [84]. Zur Häufigkeit von Nebenwirkungen auf zahnmedizinische Materialien bestehen keine wirklich belastbaren epidemiologischen Daten. Das Risiko scheint jedoch für das zahnärztliche Personal wesentlich höher zu sein als für Patienten [85]. Zur Schwere der Nebenwirkungen, wie extraorale Haut- bzw. intraorale Schleimhautreaktionen, lichenoide Veränderungen in Kontakt mit Amalgam, Goldlegierungen und Kompositkunststoffen oder marginale Gingivitis / Parodontitis, liegen ebenfalls keine umfangreichen Daten vor [86]. Es gilt jedoch zu beachten, dass es einen Unterschied zwischen dem allgemein bewerteten Risiko für einen Werkstoff und dem individuellen, den einzelnen Patienten betreffenden Risiko gibt. Deshalb stellt die Auswahl von Werkstoffen für die 


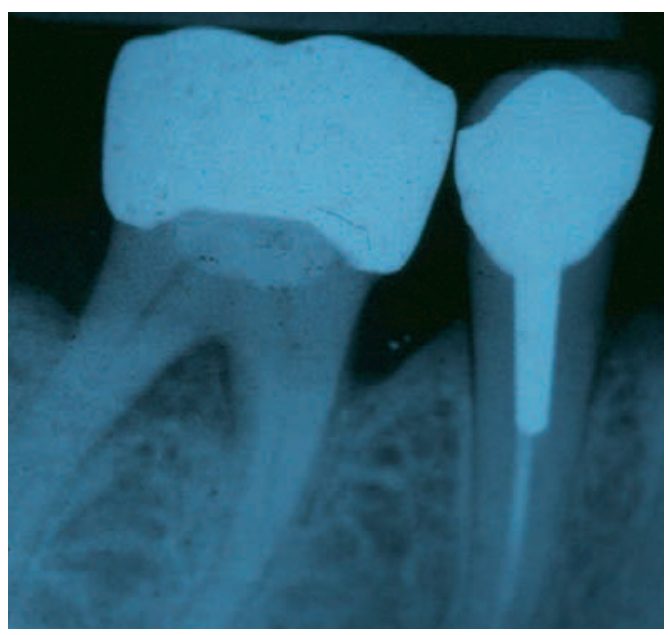

Abb. 1. Röntgenbild mit fortgeschrittenem knöchernen Attachmentverlust und Furkationsbefall an Zahn 46 .

zahnärztliche Therapie eine ernst zu nehmende Aufgabe dar, für deren Lösung allerdings kein wissenschaftlich fundiertes Procedere zur Verfügung steht [84]. Vor Entfernung und Ersatz zahnärztlicher Restaurationen sollte immer daran gedacht werden, dass diese Massnahmen mit einem erheblichen Trauma der lokalen Gewebe und des Gesamtorganismus verbunden sein können. Deshalb müssen im Einzelfall, wie das zweite Patientenbeispiel deutlich macht, Risiko und Nutzen sehr sorgfältig gegeneinander abgewogen und Art sowie Zeitpunkt von Interventionen interdisziplinär fein aufeinander abgestimmt werden.

\section{Patientenbeispiel 1:}

\section{Parodontitis und}

\section{degenerative Gelenkserkrankung}

Eine 57-jährige Patientin befand sich im Jahr 2003 wegen chronischen, weitgehend therapieresistenten Rückenbeschwerden als Folge arthrotischer Veränderungen in den Hüft- und Iliosakralgelenken beidseits sowie Spondylarthrosen der Wirbelsäule in ärztlicher Behandlung. Die schul- und komplementärmedizinischen Therapiemassnahmen erbrachten keine wesentliche Reduktion der phasenweise sehr starken Gelenk- und Muskelschmerzen.

Die durch den Arzt veranlasste konsiliarische Abklärung der Patientin durch den Zahnarzt ergab eine fortgeschrittene marginale Parodontitis im Bereich der Molaren des Ober- und Un-

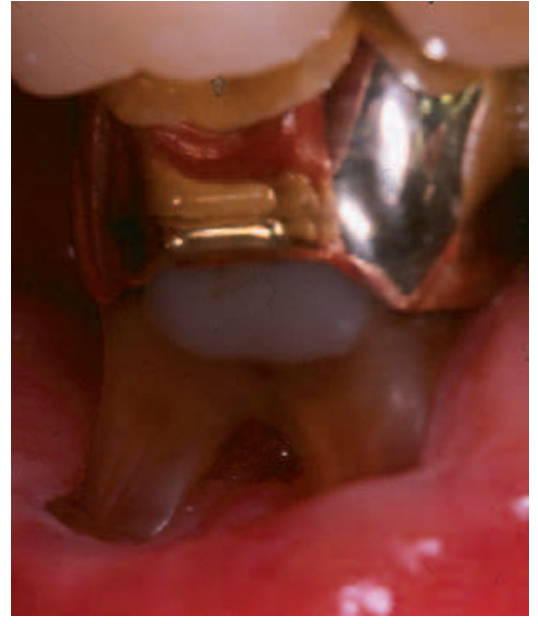

Abb. 2. Gesunde Gingiva an Zahn 46 nach Abschluss der Parodontalbehandlung.

terkiefers. Die Parodontitis hatte an den befallenen Zähnen teilweise bereits zu einem grösseren knöchernen Attachmentverlust geführt (Abb. 1). Eine negative Wirkung der parodontalen Infektion auf die Arthrosen der Wirbelsäule und Hüftgelenke konnte nicht ausgeschlossen werden, weshalb die parodontale Therapie empfohlen und auch durchgeführt wurde. Diese umfasste in einer ersten Phase die quadrantenweise durchgeführte geschlossene Behandlung der infizierten Zahnfleischtaschen innerhalb von zwei Wochen. In der nachfolgenden überwachten Heilungsphase von acht Wochen Dauer wurde die Mundhygiene der Patientin durch die lokale Anwendung eines chlorhexidinhaltigen, später eines teebaumölhaltigen Gels unterstützt. Die parodontalen Taschen, die nach diesen zwei Behandlungsphasen eine erhöhte Sondierungstiefe und eine persistierende Entzündung zeigten, wurden schliesslich chirurgisch eliminiert. Zudem wurden die Furkationen an den ersten Molaren des Unterkiefers der Hygiene mit Interdentalbürstchen zugänglich gemacht. Dank der guten Kooperation der Patientin ging nun die Entzündung des gesamten Zahnhalteapparates innerhalb weniger Wochen vollständig zurück (Abb. 2). Gleichzeitig nahmen auch die Gelenk- und Muskelschmerzen bis zur weitgehenden Beschwerdefreiheit $a b$.

Interessant war der weitere Verlauf der Patientengeschichte. Einige Mo- nate nach Abschluss der Behandlung meldete sich die Patientin notfallmässig mit starken Zahnschmerzen. Eine akute Pulpitis purulenta totalis an einem endständigen Molaren im Oberkiefer war die Ursache der Beschwerden. Mit dieser oralen Entzündung traten auch die Rückenschmerzen wieder auf. Nach erfolgreichem Abschluss der Wurzelbehandlung am Molaren bildeten sich auch diese Rückenbeschwerden innerhalb weniger Tage erneut und während der letzten fünf Jahre bleibend zurück.

\section{Patientenbeispiel 2: Werkstoffverträglichkeit und Hauterkrankung}

Eine 38-jährige Patientin wurde zu Beginn des Jahres 2000 zur Behandlung einer chronischen, am ganzen Körper auftretenden Neurodermitis stationär aufgenommen. Der Kopf und die Extremitäten waren von den grossflächigen, schweren Hautveränderungen besonders betroffen (Abb. 3). Die bis zu jenem Zeitpunkt u. a. mit Corticosteroiden durchgeführte, mehrjährige Behandlung war nicht erfolgreich und konnte eine Verschlechterung der Hauterkrankung und des Allgemeinzustandes nicht verhindern.

Die während des stationären Aufenthaltes durchgeführte komplementärmedizinische Therapie (Ernährungsumstellung, immunmodulative Massnahmen, Magen-Darmbehandlung mit Symbioselenkung, Phytotherapie und orthomolekulare Therapie) führte innerhalb weniger Wochen zu einer deutlichen Verbesserung des Allgemeinzustandes und zu einem gewissen Rückgang der Hautsymptome. Ein wirklicher Durchbruch konnte aber noch nicht erreicht werden. Es traten immer wieder, vor allem in psychischen Stresssituationen, Rezidive auf. Die Bestimmung der Art und Menge von Metallionen im Urin gab den Hinweis auf eine mögliche Belastung des Organismus vor allem mit Quecksilber. Deshalb erfolgte zusätzlich eine zahnärztliche Abklärung. In der Mundhöhle lagen multiple Amalgamfüllungen vor, die sich teilweise in einem schlechten Zustand befanden. Sie waren neben Ernährung und Umwelt als Quelle der diskutierten Quecksilberbelastung des Körpers in 


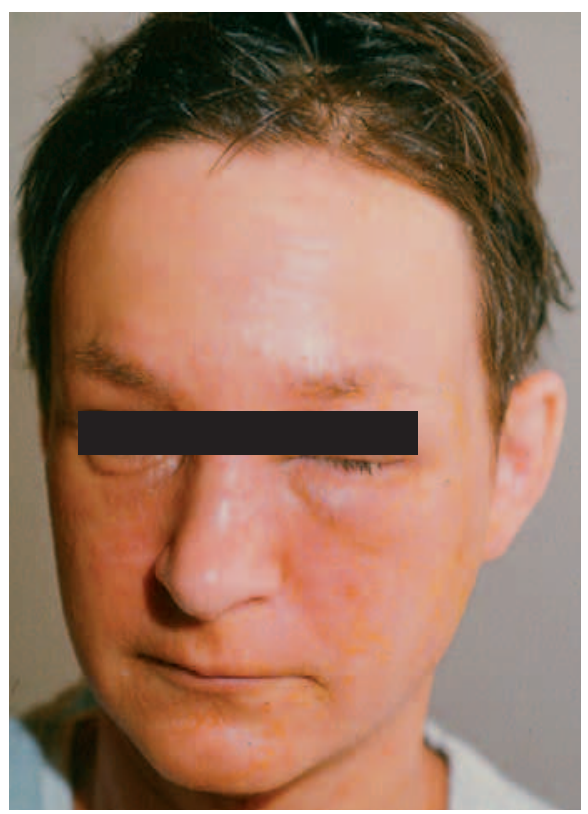

Abb. 3. Schwere Neurodermitis der Gesichtshaut. Eine bakterielle Superinfektion führte zum Zuschwellen des linken Auges.

Betracht zu ziehen. Aufgrund des bisher nur teilweisen Therapieerfolges entschloss sich die Patientin nach gründlicher Abklärung und interdisziplinärer Behandlungsplanung sowie Beratung zur Entfernung der Amalgamfüllungen und einer begleitenden medizinischen Ausleitungstherapie. Diese umfasste Selenase (1 Trinkampulle à $100 \mu \mathrm{g}$ pro Tag), Zinkorothat (40 mg pro Tag), eine klinikeigene Antioxidanzienmischung (1 gehäufter Teelöffel pro Tag, dem Essen beigemischt) sowie Succimerum (1 DMSA-Kapsel à $100 \mathrm{mg}$ pro Tag). Die Ausleitungsthera-

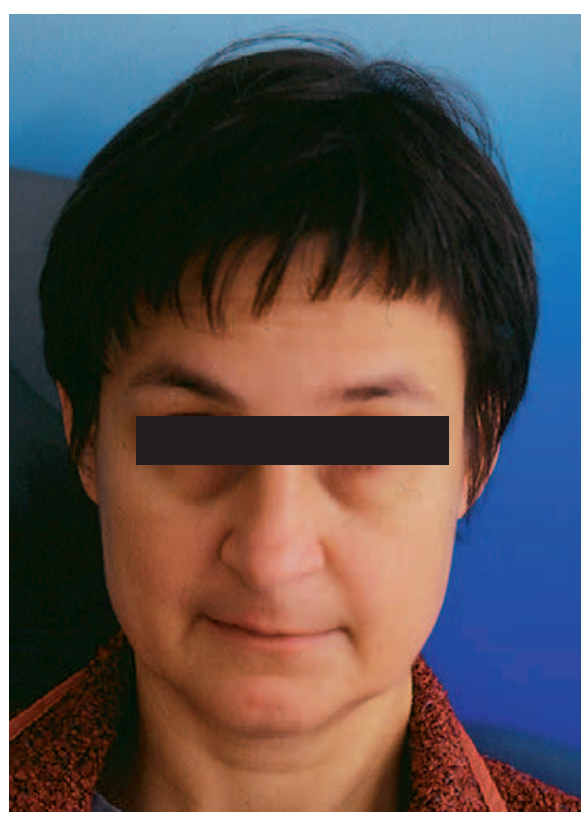

Abb. 5. Situation der Gesichtshaut sechs Jahre nach Behandlungsabschluss.

pie sollte den Organismus bei der Ausscheidung der vorhandenen Schwermetalldepots unterstützen. Stoffwechsel und Spurenelemente wurden dabei ärztlich überwacht. Nach Abschluss der Ausleitungstherapie konnte eine deutliche Reduktion der Metallbelastung im Urin nachgewiesen werden.

Die Entfernung der Amalgamfüllungen erfolgte schrittweise und unter mehrfachen Schutzmassnahmen. Pro Sitzung wurden aufgrund der Schwere der Hauterkrankung sowie der Grösse der Füllungen nur wenige Amalgamrestaurationen entfernt und mehrwö- chige Intervalle zwischen den Behandlungsterminen abgewartet, um dem Organismus jeweils eine ausreichende Erholungsphase zu bieten. Zu Behandlungsbeginn erhielt die Patientin jeweils Carbo absorbens (3 Tabletten). Die zu behandelnden Zähne wurden in der Mundhöhle mittels eines Spanngummis (Kofferdam) isoliert. Dieser verhinderte, dass die Patientin während der Amalgamentfernung Füllungspartikel verschlucken konnte. Da beim Heraustrennen von Amalgamfüllungen auch Quecksilber verdampft, wurde die Wasserkühlung während der Präparationsmassnahmen verstärkt und die Patientin erhielt zudem Sauerstoff sowie eine spezielle Nasenmaske mit Metallfilter, um die Aufnahme von Quecksilber über die Atemwege zu minimieren. Nach jeder Amalgamentfernung wurde der Mund mit N-Acetylcystein (Muco-Mepha ${ }^{\circledR} 200$ mg) gründlich ausgespült.

Der Ersatz der durchwegs im Seitenzahnbereich liegenden Amalgamfüllungen erfolgte durch nichtmetallische Werkstoffe. Kleine Kavitäten wurden mit Kompositkunststoff versorgt, grossflächige Restaurationen aus Gründen der mechanischen Festigkeit und chemischen Stabilität in Keramik gefertigt (Abb. 4). Die rekonstruktive Therapie wurde Mitte des Jahres 2001 abgeschlossen. Interessant $\mathrm{zu}$ beobachten war, dass sich die Hautsituation während der zahnärztlichen Therapie laufend verbesserte. Mit Abschluss der zahnärztlichen Behandlung wurden
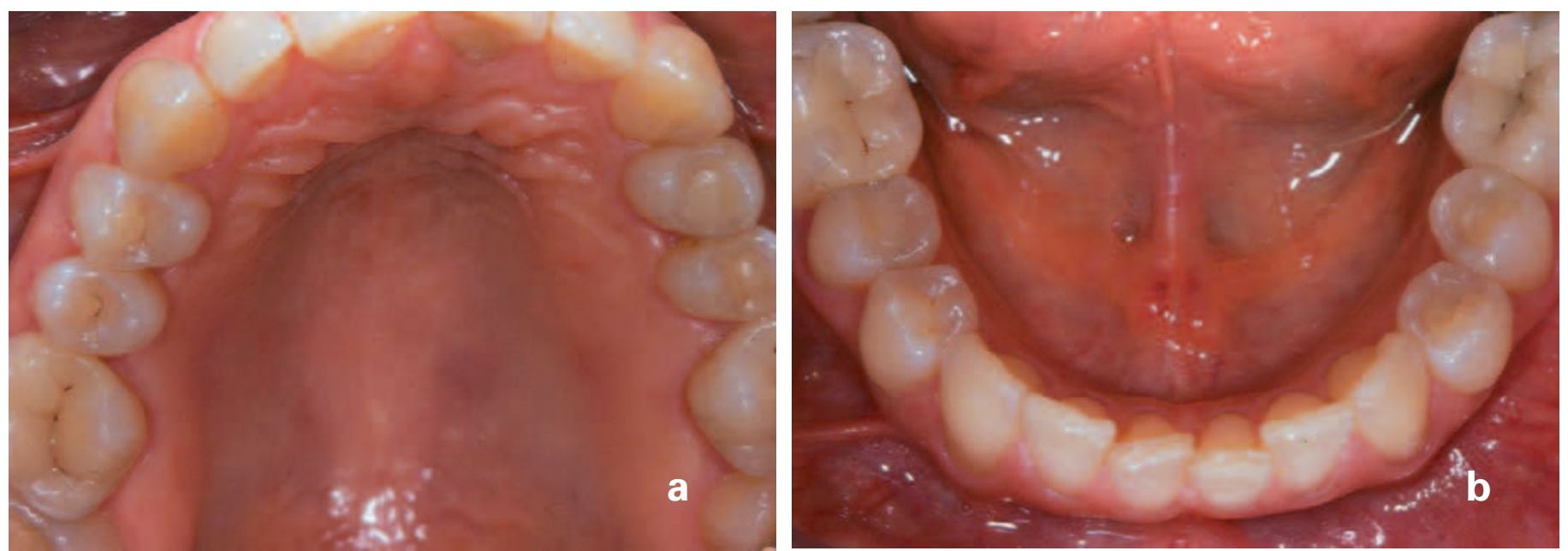

Abb. 4. Nichtmetallische Restauration der Seitenzähne im Ober- (a) und Unterkiefer (b) mit Kunststoff- und Keramikfüllungen anlässlich der Einjahreskontrolle. 
gesunde Hautverhältnisse erreicht. Dieses Behandlungsergebnis hat sich bis zur letzten Kontrolle im Jahr 2007 als stabil erwiesen (Abb. 5).

\section{Schlussfolgerungen}

Die in diesem Beitrag übersichtsweise dargestellte Vielfalt möglicher und belegter Zusammenhänge zwischen Mund- und Allgemeinerkrankungen unterstreicht insbesondere für den alternden Patienten mit zunehmenden systemischen Defiziten und einer in der Regel längeren zahnmedizinischen Vorgeschichte die dringende Notwendigkeit einer interdisziplinären Beurteilung der zahn- und allgemeinmedizinischen Befunde und Diagnosen. Möglicherweise notwendig werdende medizinische und zahnärztliche Therapiemassnahmen sollten ebenfalls aufeinander abgestimmt werden.

\section{Literatur}

1 Siepmann M, Kirch W: Multimorbidität Interaktionen von Zahn- und allgemeinen Erkrankungen. Quintessenz 2002;53:391-403.

2 Imfeld Th: Allgemeinmedizinische Bedeutung der oralen Gesundheit. Acta Med Dent Helv 2000;5:49-50

3 Dörfer Ch: Einfluss der Parodontitis auf die Allgemeinerkrankungen. Quintessenz 2007;58: 1193-1200.

4 Van Winkelhoff AJ, Overbeek BP, Pavicic MJ, van den Bergh JP, Ernst JP, de Graaff J: Longstanding bacteremia caused by oral Actinobacillus actinomycetemcomitans in a patient with a pacemaker. Clin Infect Dis 1993;16: 216-218.

5 DeBowes LJ: The effects of dental disease on systemic disease. Vet Clin North Am Small Anim Pract. 1998;28:1057-1062.

6 Beck JD, Offenbacher S: Orale Gesundheit und systemische Erkrankungen: Parodontitis und kardiovaskuläre Erkrankungen. Acta Med Dent Helv 2000;5:56-68.

7 Kohal RJ, Lutter G, Dennison DK: Marginale Parodontitis und kardiovaskuläre Erkrankungen. Schweiz Monatsschr Zahnmed 2001;111: 445-450.

8 Li X, Kolltveit KM, Tronstad L, Olsen I: Systemic diseases by oral infection. Clin Microbiol Rev 2000;13:547-558.

9 Madianos PN, Bobetsis GA, Kinane DF: Is periodontitis associated with an increased risk of coronary heart disease and preterm and/or low birth weight births? J Clin Periodontol 2002;29, Suppl 3:22-36

10 Holmstrup P, Poulsen AH, Andersen L, Skuldbol T, Fiehn NE: Oral infections and systemic diseases. Dent Clin North Am 2003;47:575598.

11 Janket SJ, Baird AE, Chuang SK, Jones JA: Meta-analysis of periodontal disease and risk of coronary heart disease and stroke. Ora Surg Oral Med Oral Pathol Oral Radiol Endod 2003;95:559-569.
12 Elter JR, Champagne CME, Offenbacher S: Relationship of periodontal disease and tooth loss to prevalence of coronary heart disease. J Periodontol 2004;75:782-790.

13 Pussinen PJ, Alfthan G, Rissanen H: Antibodies to periodontal pathogens and stroke risk. Stroke 2004;35:2020-2023.

14 Ragnarsson E, Eliasson ST, Gudnason V: Loss of teeth and coronary heart disease. Int $J$ Prosthodont 2004;17:441-446.

15 Engebretson SP, Lamster IB, Elkind MSV: Radiographic measures of chronic periodontitis and carotid artery plaque. Stroke 2005;36: 561-566.

16 Montebugnoli M, Servidio D, Miaton RA: Periodontal health improves systemic inflammatory and haemostatic status in subjects with coronary heart disease. J Clin Periodontol 2005;32:188-192.

17 Bahekar AA, Singh S, Saha S, Molnar J, Arora $R$ : Possible link between periodontal disease and coronary heart disease. Am Heart J 2007; 154:830-837.

18 Hujoel PP, Drangsholt M, Spiekerman C, DeRouen TA: Periodontal disease and coronary heart disease risk. JAMA 2000;284:14061410.

19 Hujoel PP, Drangsholt M, Spiekerman C, DeRouen TA: Examing the link between coronary heart disease and the elimination of chronic dental infections. J Am Dent Assoc 2001;132:883-889.

20 Jansson L, Lavstedt S, Frithiof L, Theobald H: Relationship between oral health and mortality in cardiovascular diseases. J Clin Periodontol $2001 ; 28: 762-768$

21 Joshipura KJ, Wand HC, Merchant AT, Rimm EB: Periodontal disease and biomarkers related to cardiovascular disease. J Dent Res 2004; 83:151-155

22 Lösche W: Marginale Parodontitis und HerzKreislauf-Erkrankungen. Quintessenz 2004; 55:393-402.

23 Mercado FB, Marshall RI, Klestov A, Bartold PM: Is there a relationship between rheumatoid arthritis and periodontal disease? J Clin Periodontol 2000;27:267-272

24 Mercado FB, Marshall RI, Bartold PM: Interrelationships between rheumatoid arthritis and periodontal disease: a review. J Clin Periodontol 2003;30:761-772.

25 Emrich LJ, Schlossman M, Genco RJ: Periodontal disease in non-insulin dependent diabetes mellitus. J Periodontol 1991;62:123.

26 Löe H: Periodontal disease. The sixth complication of diabetes mellitus. Diabetes Care 1993;16:329.

27 Grossi SG, Zambon JJ, Ho AW, Koch G, Dunford RG, Machtei EE, Norderyd OM, Genco RJ: Assessment of risk for periodontal disease. I. Risk indicators for attachment loss. J Periodontol 1994;65:260-267.

28 Grossi SG, Genco RJ: Periodontal disease and diabetes mellitus: A two-way relationship. Ann Periodontol 1998;3:51-61.

29 Grossi SG: Parodontale Erkrankungen und Diabetes mellitus: Eine wechselseitige Beziehung. Acta Med Dent Helv 2000;5:51-55.

30 Taylor GW, Loesche WJ, Terpenning MS: Impact of oral diseases on systemic health in the elderly: diabetes mellitus and aspiration pneumonia. J Public Health Dent 2000; 60:313-320

31 Soskolne WA, Klinger A: The relationship between periodontal diseases and diabetes: an overview. Ann Periodontol 2001;6:91-98.

32 Shay K: Infectious complications of dental and periodontal diseases in the elderly population. Clin Infect Dis 2002:34:1215-1223.
33 Ruppert M, Berres F, Marinello CP: Aggressive generalisierte schwere Parodontitis und Brittle Diabetes mellitus Typ I. Schweiz Monatsschr Zahnmed 2003;113:533-544.

34 Khader YS, Dauod AS, El-Qaderi SS, Alkafajei A, Batayha WQ: Periodontal status of diabetics compared with nondiabetics: a meta-analysis. J Diabetes Complications 2006;20:59-68.

35 Bender IB, Bender AB: Diabetes mellitus and the dental pulp. J Endodont 2003;29:383-389

36 Seppala B, Ainamo J: A site-by-site follow-up study on the effect of controlled versus poorly controlled insulin-dependent diabetes mellitus. J Clin Periodontol 1994;21:161-165.

37 Tervonen T, Karjalainen K: Periodontal disease related to diabetic status. A pilot study of the response to periodontal therapy in type 1 diabetes. J Clin Periodontol 1997;24:505-510.

38 Taylor GW, Burt BA, Becker MP, Genco RJ, Schlossman M: Glycemic control and alveolar bone loss progression in type 2 diabetes. Ann Periodontol 1998;3:30-39.

39 Lalla E, Park DB, Papapanou PN, Lamster IB Oral disease in northern Manhattan patients with diabetes mellitus. Am J Public Health 2004;94:755-758

40 Salvi GE, Kandylaki M, Troendle A, Persson GR, Lang NP: Experimental gingivitis in type 1 diabetics: a controlled clinical and microbiolog ical study. J Clin Periodontol 2005;32:310-316.

41 Rodrigues DC, Taba MJ, Novaes AB, Souza SL, Grisi MF: Effect of non-surgical periodontal therapy on glycemic control in patients with type 2 diabetes mellitus. J Periodonto 2003;74:1361-1367

42 Kiran M, Arpak N, Unsal E, Erdogan MF: The effect of improved periodontal health on metabolic control in type 2 diabetes mellitus. J Clin Periodontol 2005;32:266-272

43 Promsudthi A, Pimapansri S, Deerochanawong C, Kanchanavasita W: The effect of periodontal therapy on uncontrolled type 2 diabetes mellitus in older subjects. Oral Dis 2005:11:293-298.

44 Aldridge JP, Lester V, Watts TL, Collins A, Viberti G, Wilson RF: Single-blind studies of the effects of improved periodontal health on metabolic control in type 1 diabetes mellitus $\mathrm{J}$ Clin Periodontol 1995;22:271-275.

45 Janket SJ, Wightman A, Baird AE, Van Dyke $T E$, Jones JA: Does periodontal treatment improve glycemic control in diabetic patients? A meta-analysis of intervention studies. Dent Res 2005;84:1154-1159.

46 Grossi SG, Skrepcinski FB, DeCaro T, Robertson DC, Ho AW, Dunford RG, Genco RJ: Treatment of periodontal disease in diabetics reduces glycated haemoglobin. J Periodontol 1997;68:713-719.

47 Terpenning $M$, Bretz $W$, Lopatin D, Langmore $\mathrm{S}$, Dominguez $\mathrm{B}$, Loesche W: Bacterial colonization of saliva and plaque in the elderly. Clin Infect Dis 1993;16:314-316.

48 Mojon P, Budtz-Jörgensen E, Michel JP, Limeback $\mathrm{H}$ : Oral health and history of respiratory tract infection in frail institutionalized elders. Gerodontol 1997;14:9-16.

49 Russell SL, Boylan RJ, Kaslick RS, Scannapieco FA, Katz RV: Respiratory pathogen colonization of the dental plaque of institutionalized elders. Spec Care Dent 1999;19:1-7.

50 Scannapieco FA: Orale Erkrankungen und Infektionen der Atemwege. Acta Med Dent Helv 2000:5:74-77.

51 Yoneyama T, Yoshida M, Ohrui T, Mukaiyama H, Okamoto H, Hoshiba K, Ihara S, Yanagisawa S, Ariumi S, Morita T, Mizuno Y, Ohsawa T, Akagawa Y, Hashimoto K, Sasaki H: Oral care reduces pneumonia in older patients in nursing homes. J Am Geriatr Soc 2002;50: 430-433. 
52 Okuda K, Kimizuka R, Abe S, Kato T, Ishihara $\mathrm{K}$ : Involvement of periodontopathic anaerobes in aspiration pneumonia. J Periodontol 2005; 76:2154-2160.

53 Azarpashooh A, Leake JL: Systematic review of the association between respiratory diseases and oral health. J Periodontol 2006;77: 1465-1482.

54 Reinhardt RA, Payne JB, Maze CA, Patil KD, Gallagher SJ, Mattson JS: Influence of estrogen and osteopenia/osteoporosis on clinical periodontitis in postmenopausal women. J Periodontol 1999;70:823-828.

55 Wactawski-Wende J: Periodontal diseases and osteoporosis: association and mechanisms. Ann Periodontol 2001;6:197-208.

56 Reddy MS: Oral osteoporosis: is there an association between periodontitis and osteoporosis. Compend Contin Educ Dent 2002;23, Suppl10:21-28.

57 Mohammad AR, Hooper DA, Vermilyea SG, Mariotti $A$, Preshaw PM: An investigation of the relationship between systemic bone density and clinical periodontal status in postmenopausal Asian-American women. Int Dent J 2003;53:121-125.

58 Shen EC, Gau CH, Hsieh YD, Chang CY, Fu E: Periodontal status in post-menopausal osteoporosis: a preliminary clinical study in Taiwanese women. J Chin Med Assoc 2004;67:38993.

59 Yoshihara A, Seida Y, Hanada N, Miyazaki H: A longitudinal study of the relationship between periodontal disease and bone mineral density in community-dwelling older adults. J Clin Periodontol 2004;31:680-684.

60 Dervis E: Oral implications of osteoporosis. Oral Surg Oral Med Oral Pathol Oral Radiol Endod 2005;100:349-356.

61 Inagaki K, Kurosu Y, Yoshinari N, Noguchi T, Krall EA, Garcia RI: Efficacy of periodontal disease and tooth loss to screen for low bone mineral density in Japanese women. Calcif Tissue Int 2005;77:9-14.

62 Lerner UH: Inflammation-induced bone remodelling in periodontal disease and the influence of post-menopausal osteoporosis. J Dent Res 2006;85:596-607.

63 Kato T, Usami T, Noda Y, Hasegawa M, Ueda $M$, Nabeshima 'T: The effect of the loss of molar teeth on spatial memory and acetylcholine release from the parietal cortex in aged rats. Behav Brain Res 1997;83:239-242.

64 Yoshida M, Morikawa $H$, Kanehisa Y, Taji T, Tsuga K, Ákagawa Y: Functional dental occlusion may prevent falls in elderly individuals with dementia. J Am Geriatr Soc 2005;53: 1631-1632.

65 Deinzer R, Rüttermann S, Möbes O, Herfordt $A$ : Increase in gingival inflammation under academic stress. J Clin Periodontol 1998;25:431433.

66 Krahwinkel Th, Boekstegen C: Zusammenhang zwischen Stressparametern und Parodontopathien. Quintessenz 2003;54:169-177.

67 Vettore M, Quintanilha RS, Monteiro da Silva AM, Lamarca GA, Leão AT: The influence of stress and anxiety on the response of non-surgical periodontal treatment. J Clin Periodontol 2005;32:1226-1235.

68 Lang H: Parodontitis durch Stress?! Dtsch Zahnärztl Z 2007;62:134-136

69 Jeffcoat MK: Parodontale Erkrankung: Risikofaktor für Frühgeburten? Acta Med Dent Helv 2000;5:69-73

70 Jeffcoat MK, Geurs NC, Reddy MS, Cliver SP, Goldenberg RL, Hauth JC : Periodontal infection and preterm birth: Results of a prospective study. J Am Dent Assoc 2001;132:875880 .
71 López NJ, Smith PC, Gutierrez J: Periodontal therapy may reduce the risk of preterm low birth weight in women with periodontal disease: a randomized controlled trial. J Periodontol 2002;73:911-924

72 Khader YS, Ta'ani Q: Periodontal diseases and the risk of preterm birth and low birth weight: a meta-analysis. J Periodontol 2005;76:161165

73 Moreu G, Téllez L, González-Jaranay M: Relationship between maternal periodontal disease and low-birth-weight pre-term infants. $\mathrm{J}$ Clin Periodontol 2005;32:622-627.

74 Vergnes J-N, Sixou M: Preterm low birth weight and maternal periodontal status: a metaanalysis. Am J Obstet Gynecol 2007; 196:135e1-135e7.

75 Marques da Silva R, Caugant DA, Josefsen R, Tronstad L, Olsen I: Characterization of Streptococcus constellatus strains recovered from a brain abscess and periodontal pockets in an immunocompromised patient. J Periodontol 2004;75:1720-1723

76 Strojnik T, Roskar Z: Brain abscess after milk tooth self-extraction. Wien Klin Wochenschr 2004;116 Suppl2:87-89.

77 Ewald C, Kuhn S, Kalff R: Pyogenic infections of the central nervous system secondary to dental affections - a report of six cases. Neurosurg Rev 2006;29:163-166.

78 Crippin JS, Wang KK: An unrecognized etiology for pyogenic hepatic abscesses in normal hosts: dental disease. Am J Gastroenterol 1992;87:1740-1743.

79 Schiff E, Pick N, Oliven A, Odeh M: Multiple liver abscesses after dental treatment. J Clin Gastroenterol 2003;36:369-371.

80 Wagner KW: Hirn- und Leberabszesse durch Streptokokkus intermedius. ZM 2006;96:252254.

81 Dudeney TP, Todd IP: Crohn's disease of the mouth. Proc Soc Med 1969:62:1237.

82 Tiedemann Ch, Wetzel A: Parodontitis als Manifestation einer Systemerkrankung. Schweiz Monatsschr Zahnmed 2001;111: 1091-1097.

83 Siepmann M, Kirch W: Medizinische Befunde, Alter, Risiko. Dtsch Zahnärztl Z 2007:62:6-14.

84 Schmalz G, Garhammer P: Wenn der Werkstoff zum Risiko wird - Diagnostik und Wechselwirkungen. Quintessenz 2007;58:11631168.

85 Kallus T, Mjör IA: Incidence of adverse effects of dental materials. Scand J Dent Res 1991; 99:236-240

86 Garhammer P, Schmalz G, Hiller KA, Reitinger T. Stolz W: Patients with local adverse effects from dental alloys: frequency, complaints, symptoms allergy. Clin Oral Invest 2001;5: 240-249.

\section{Disclosure Statement}

The author declares that no financial or other conflict of interest exists in relation to the content of this article.

\section{Korrespondenzadresse}

Prof. Dr. med. dent. Christian E. Besimo Aeskulap-Klinik / Abteilung Zahnmedizin Gersauerstr. 8, CH-6440 Brunnen

christian.besimo@aeskulap.com 